\title{
Clinicopathological characteristics and oncological outcomes of patients with renal cell carcinoma
}

\author{
Madura Ambegoda ${ }^{1}$, Rakitha Paranamanna ${ }^{1}$, Sagara Kumara ${ }^{1}$, Balasingam Balagobi ${ }^{1}$, Cherine \\ Sosai ${ }^{2}$, Arunasalam Pathmeswaran ${ }^{3}$, Anuruddha Abeygunasekera ${ }^{1}$
}

(Index words: renal cancer, prognosis, south Asia, Sri Lanka, survival data, renal cell carcinoma)

\begin{abstract}
Although certain characteristics of renal cell carcinoma (RCC) seen in Sri lanka are different to the developed world and incidence is rising, no data on oncological outcome and patient survival are avaialble. A hospitalbased cohort study was done in a tertiary referral centre over a period of eight years (2012-2019). A total of 285 newly diagnosed RCC had a mean follow-up period of 28.3 months. Radical nephrectomy was performed in 189 patients while 91 patients had partial nephrectomy. Clear cell RCC which was seen in 231 (81.1\%) patients while papillary RCC was seen in 43 (15.1\%). There were 54 deaths during the follow-up. The 30 -day postoperative mortality was $2.1 \%$. Thirty $(10.5 \%)$ patients developed metastases while three patients developed local recurrences. The overall five-year survival rate was $72.8 \%$ (95\% Cl=65.9-80.5). Five-year survival of papillary RCC (60.5\%) was lower than clear cell RCC (74.1\%). Tumour stage, presence of metastases and histological grade of the tumour influenced the survival. Gender $(p=0.3)$, histological type $(p=0.5)$ or type of surgery $(p=0.2)$ had no statistically significant effect on survival.
\end{abstract}

Ceylon Medical Journal 2020; 65: 62-66

DOI: http://doi.org/10.4038/cmj.v65i3.9187

\section{Introduction}

Renal cancer accounts for $5 \%$ of all cancers in men and $3 \%$ of all cancers in women [1]. Worldwide, renal cancer is the 13th most common malignancy, with approximately 338000 new cases diagnosed in each year. Renal cell carcinoma (RCC) accounts for $90 \%$ of all renal malignancies. The incidence of RCC varies geographically. Rates of RCC are highest in Europe, North America, and Australia, whereas rates are low in India, Japan, Africa, and China. Although an increase in RCC has been observed globally, in recent years incidence of RCC has declined in some European countries namely Sweden, Poland, Finland and Netherlands. The crude incidence of renal cancer in Sri Lanka is 1.3 per 100000 population according to latest available statistics of National Cancer Registry for the year 2014 in which 255 new cases of RCC had been reported [2]. In 2010, only 169 cases of renal cancer were reported to the National Cancer Registry [3].

The characteristics and behaviour of RCC differs in different countries. Average age at the time of diagnosis of RCC in Sri Lankan patients is lower than the developed world with a large proportion (27.5\%) of patients being under 50 years [4]. There are no publications related to outcome of surgery which is the mainstay of treatment for RCC and long-term survival of patients with RCC in Sri Lanka [5]. The main objective of our study was to determine the survival data of patients with RCC.

\section{Methods}

This was a hospital based prospective cohort study. In the urology unit of Colombo South Teaching Hospital (CSTH), a comprehensive cancer registry and a data base is maintained. Adult ( $\geq 18$ years) patients who had treatment for histologically confirmed renal cell carcinoma in the urology unit, CSTH from 1 January 2012 to 31 December 2019 were eligible to be included in the study. They were followed up after appropriate treatment. The data related to demography, clinical characteristics, types of treatment and findings during follow-up visits were recorded prospectively in a data sheet. The duration of the follow-up was calculated from date of diagnosis to the date of death or last follow-up. The primary end point of this study was overall survival. Follow-up assessment ended on 30 April 2020.

${ }^{1}$ Department of Urology, Colombo South Teaching Hospital, Dehiwala, Sri Lanka, ${ }^{2}$ Department of Pathology, Colombo South Teaching Hospital, Dehiwala, Sri Lanka, ${ }^{3}$ Department of Public Health, Faculty of Medicine, University of Kelaniya, Ragama, Sri Lanka.

Correspondence: AMA, e-mail: <amabey@sltnet.Ik>. Received 17 May 2020 and revised version 28 July 2020 accepted 20 October 2020.

This is an open-access article distributed under the terms of the Creative Commons Attribution License, which permits unrestricted use, distribution, and reproduction in any medium, provided the original author and source are credited. 
All patients with renal tumours had a CECT KUB if serum creatinine was below $150 \mu \mathrm{mol} / \mathrm{l}$ to characterise the lesion. Depending on the RENAL nephrometry score [6], age, existing comorbidities, advice of oncologists and patient's wish, decisions were taken to perform partial or radical nephrectomy. Those who were selected for focal therapy (radio-frequency ablation or microwave thermal ablation) or were not fit enough for surgery or opted to have other forms of treatment had ultrasound guided core biopsy to confirm the histology. Histopathological evaluation was done according to the World Health Organisation (WHO) and International Society of Urological Pathology (ISUP) classification, 2016 [7]. Tumour grading was based on the Fuhrman grading system initially and the ISUP grading system subsequently after its development. Since the differences between the two grading systems were minimal comparable grades were used interchangeably. Tumour staging was done using the TNM classification of the Union for International Cancer Control 2009.

Primary endpoint was overall survival time, which was defined as the time from diagnosis until death or until the date of last contact for living patients. For statistical analysis, survival rates were calculated using the KaplanMeier methods and p value $<0.05$ was taken as significant. Approval for the study was obtained from the Ethics Review Committee of the Institute.

\section{Results}

During the study period, 352 patients with renal tumours were treated in the unit. There were 285 patients with histologically confirmed renal cell carcinoma. These 285 patients constituted the study sample. Among the patients with RCC, there were 224 men with a male to female ratio of 3.7:1 (Table 1). Mean age of patients with RCC was 56.6 years $(\mathrm{SD}=12.2)$. Table 1 shows the age distribution of the patients and 82 (28.8\%) were below the age of 50 years. Distribution of the RCC according to histological type is given in Table 1 . There were eight patients with bilateral multiple renal cell carcinoma. Seven were clear cell RCC and one was a papillary RCC. Six of eight met criteria for the diagnosis of von Hippel-Lindau syndrome.

Surgery was performed in 280 patients (Table 2). Radical nephrectomy was performed in 189 patients while 91 patients had partial nephrectomy. Two patients had focal therapy only (microwave ablation), two patients had symptomatic treatment only and one patient had tyrosine kinase inhibitor - sorafenib as the primary treatment for varying reasons. Five patients with bilateral tumours had focal therapy in addition to surgery. The other three patients with bilateral tumours had bilateral surgery.

Distribution of the tumour stage and grade as determined after histopathological examination and appropriate investigation work up is given in table 1 . The commonest histological type was clear cell RCC which was seen in 231 (81.1\%) patients while papillary RCC was seen in $43(15.1 \%)$ patients. ISUP grade 2 was the commonest and was seen in 128 (44.9\%) patients. Majority (56.8\%) of patients belonged to the T1 stage. Thirty-five (12.3\%) patients had metastases at the time of diagnosis. Bone was the commonest site of metastases $(n=13)$ while lungs $(n=11)$, liver $(n=8)$ and adrenal glands $(n=7)$ followed. In four out of seven patients with adrenal metastases, the preoperative CT urogram raised suspicion and in the remaining three patients, intraoperative palpation suggested adrenal masses.

Tumour was extending to the renal vein in 41 (14.4\%) patients and confirmed histologically. In fourteen (4.9\%) of them the tumour thrombus was extending to the IVC. Two of them had supradiaphragmatic extensions necessitating synchronous open heart surgery.

In our series the mean follow-up period was 28.3 months $(S D=24.8$, median $=21$ months, range 6-96 months). There were 54 deaths during the follow-up. Six of them died within 30 days of surgery leading to a postoperative mortality of $2.1 \%$. Thirty $(10.5 \%)$ patients developed metastases during the follow-up period. Three patients developed local recurrences (two after partial nephrectomy and one after radical nephrectomy) and one of them had systemic metastases in the liver also at that time. Two of them had sarcomatoid component in the initial histology and the other was with papillary type 2 RCC but presented with a perinephric haematoma after tumour rupture. Nine patients died of causes unrelated to the treatment or the progression of the disease.

The overall five-year survival rate was 72.8\% (95\% $\mathrm{CI}=65.9-80.5)$. Advancing tumour stage, presence of metastases at the time of diagnosis and ISUP grade 4 had a significant negative effect on the survival (Table 2). The gender did not have a statistically significant influence on survival, but females have a better five-year survival of $78.6 \%$ compared to men (71.3\%). Clear cell RCC had a five-year survival of 74.1\% (95\% CI=66.6-82.5) while papillary RCC had a five-year survival of 61.5\% (95\% $\mathrm{CI}=42.8-88.2$ ), but the difference was not statistically significant (Figure 1). All eight patients with chromophobe RCC were alive at the end of the study period. One patient who had multi-cystic renal tumour of low malignant potential died of a myocardial infarction while others were alive at the end of the follow-up. The five-year survival rates for radical nephrectomy and partial nephrectomy were 70.5\% (95\% CI=62.1-79.9) and 79.9\% (95\%=68.593.2) with a p value of 0.2 (Figure 1 ). 
Table 1. Characteristics of patients with renal cell carcinoma

\begin{tabular}{|c|c|}
\hline Characteristic & Number (\%) \\
\hline \multicolumn{2}{|l|}{ Sex $(3.7: 1)$} \\
\hline Male & $224(78.6)$ \\
\hline Female & $61(21.4)$ \\
\hline \multicolumn{2}{|l|}{ Age } \\
\hline$<50$ years & $82(28.8)$ \\
\hline 51-69 years & $168(58.9)$ \\
\hline$>70$ years & $35(12.3)$ \\
\hline \multicolumn{2}{|l|}{$\mathrm{T}$ stage } \\
\hline $\mathrm{T} 1$ & $162(56.8)$ \\
\hline $\mathrm{T} 2$ & $45(15.8)$ \\
\hline Т 3 & $68(23.9)$ \\
\hline $\mathrm{T} 4$ & $10(3.5)$ \\
\hline \multicolumn{2}{|l|}{$\mathrm{N}$ stage } \\
\hline N1 & 1 \\
\hline N2 & 6 \\
\hline Metastases & $35(12.3)$ \\
\hline Bone & 13 \\
\hline Lung & 10 \\
\hline Liver & 8 \\
\hline Adrenal gland & 7 \\
\hline \multicolumn{2}{|l|}{ Treatment } \\
\hline Radical nephrectomy & $189(66.3)$ \\
\hline Partial nephrectomy & 91 (31.9) \\
\hline Focal therapy only & $2(0.5)$ \\
\hline No specific treatment & $2(0.5)$ \\
\hline Tyrosine kinase inhibitors only & $1(0.2)$ \\
\hline \multicolumn{2}{|l|}{ Histological type } \\
\hline Clear cell renal cell carcinoma & $231(81.1)$ \\
\hline Papillary renal cell carcinoma & $43(15.1)$ \\
\hline Chromophobe renal cell carcinoma & $8(2.8)$ \\
\hline $\begin{array}{l}\text { Multilocular cystic clear cell renal neoplasm of } \\
\text { low malignant potential }\end{array}$ & $3(1.1)$ \\
\hline \multicolumn{2}{|l|}{ ISUP grade } \\
\hline 1 & $50(17.5)$ \\
\hline 2 & $128(44.9)$ \\
\hline 3 & $51(17.9)$ \\
\hline 4 & $21(7.4)$ \\
\hline Not available & 35 (12.3) \\
\hline Total & 285 \\
\hline
\end{tabular}


Table 2. Factors influencing survival

\begin{tabular}{|c|c|c|c|}
\hline & Five-year survival (\%) & $95 \% C I$ & $p$ value \\
\hline Overall survival & 72.8 & $65.9-80.5$ & \\
\hline \multicolumn{4}{|l|}{ Gender } \\
\hline Female & 78.6 & $63.8-96.8$ & \\
\hline Male & 71.3 & $63.6-80$ & 0.3 \\
\hline \multicolumn{4}{|l|}{ Nephrectomy } \\
\hline Radical & 70.5 & 62.1-79.9 & \\
\hline Partial & 79.9 & $68.5-93.2$ & 0.3 \\
\hline \multicolumn{4}{|l|}{ Histology } \\
\hline Clear cell & 74.1 & $66.6-82.5$ & \\
\hline Papillary & 60.5 & $42.8-88.2$ & 0.6 \\
\hline \multicolumn{4}{|l|}{ Tumour stage } \\
\hline $\mathrm{T}_{1}$ & 81.1 & $72.7-90.4$ & \\
\hline $\mathrm{T}_{2}$ & 76.5 & $61.2-95.5$ & \\
\hline $\mathrm{T}_{3}$ & 54.7 & 39.3-75.9 & \\
\hline $\mathrm{T}_{4}$ & 35.6 & 12.7-99.4 & 0.001 \\
\hline \multicolumn{4}{|l|}{ Metastases } \\
\hline $\mathrm{M}_{0}$ & 77.4 & $70.3-85.2$ & \\
\hline $\mathrm{M}_{1}$ & 16 & $3.2-78.5$ & 0.001 \\
\hline \multicolumn{4}{|l|}{ ISUP grade } \\
\hline 1 & 87.9 & $77.2-99$ & \\
\hline 2 & 74.2 & $64.6-85.1$ & \\
\hline 3 & 48.4 & 25.1-93.2 & \\
\hline 4 & 42.9 & 19.9-92.1 & 0.001 \\
\hline
\end{tabular}

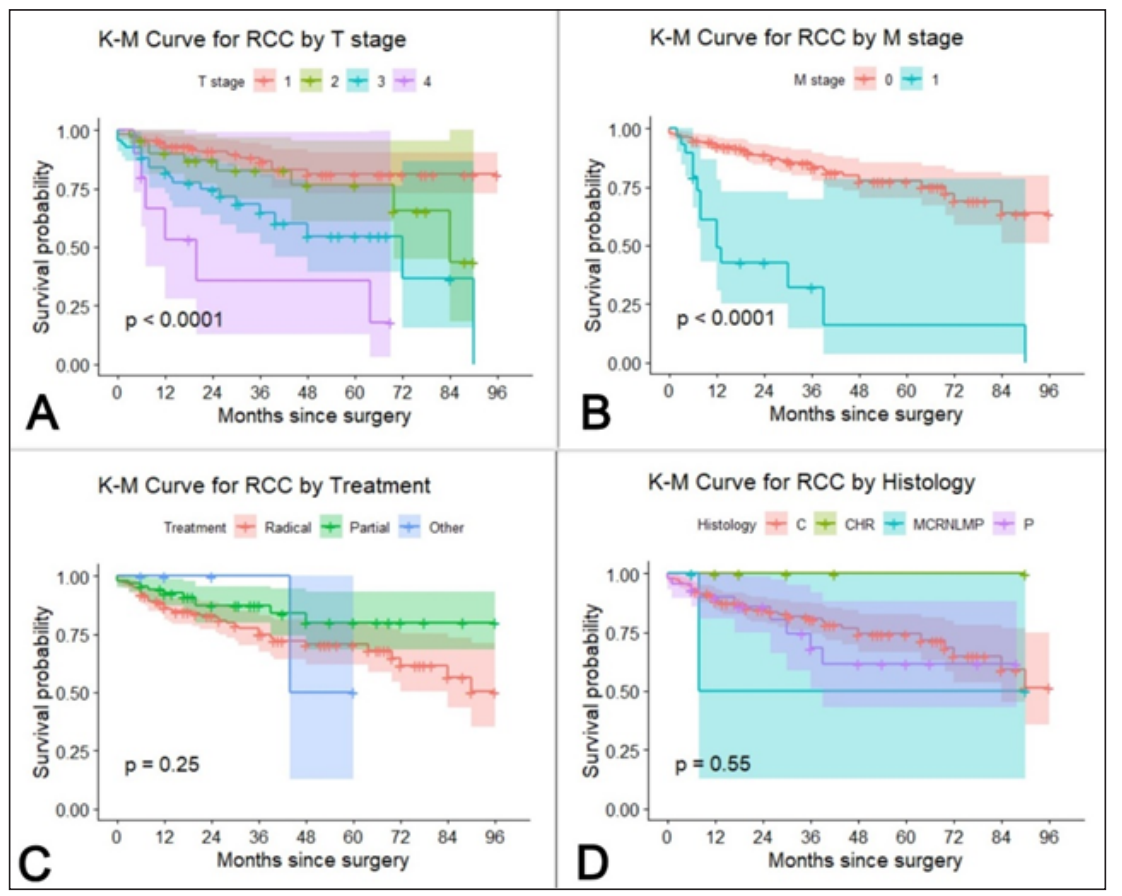

Figure 1. Overall survival stratified by A. tumour stage (B) presence of metastases (C) type of treatment and (D) histological type (C- clear cell RCC, P-papillary RCC, CHR-chromophobe RCC, MCRNLMP-multi-cystic renal neoplasm of low malignant potential). 


\section{Discussion}

The patients with renal cell carcinoma included in this study from Sri Lanka differs from international cohorts by the higher male to female ratio. The overall five-year survival of RCC in this cohort (72.8\%) is lower than the developed world but better than the neighbouring India. The five-year survival for clear cell RCC is $85.1 \%$, 88.6\% and $74.1 \%$ in Germany, Japan and in our study respectively $[8,9]$. In India the five-year survival after radical nephrectomy for RCC is 56.1\% [10].

The proportion of patients with papillary RCC is higher in our study (15.1\%) compared to that of Europe (10\%-12\%), India (9.8\%) and Japan (5\%) [8,9,10]. Generally, the prognosis of papillary RCC is considered better than clear cell RCC [11]. However, in this study the five-year survival of papillary RCC (60.5\%) is lower than clear cell RCC (74.1\%) although the difference did not reach statistical significance.

The distribution of tumour $(\mathrm{T})$ stage and ISUP grade of patients in this study are similar to the pattern in the developed world. $\mathrm{T}_{1}$ and $\mathrm{T}_{3}$ are the common tumour size groups while ISUP 2 is the common grade of the RCC. Rate of renal vein (14.4\%) and inferior vena cava (4.9\%) involvement by the tumour thrombus in our study is similar to worldwide figures $15 \%$ and $4-8 \%$ respectively [12].

Only 35 (12.3\%) patients in our study had evidence of metastases at the time of the diagnosis. This is much less than the neighbouring India where the rate of metastases at the time of diagnosis is around 40\% [10]. The proportion of patients with metastases at the time of diagnosis in our study is similar to that of the developed world which is about $10 \%$ [11]. Rate of adrenal metastases was only $2.5 \%$ and could be diagnosed by CT evaluation or intraoperative palpation with success. Hence the traditional approach of removing the adrenal gland during radical nephrectomy based on the recommendations of a post-mortem study should be abandoned [13]. Adrenalsparing radical nephrectomy can be recommended for locally advanced tumours with no evidence of adrenal involvement in CT scan or intraoperative palpation.

The difference in survival between partial nephrectomy $(79.9 \%)$ and radical nephrectomy $(70.5 \%)$ is not statistically significant at five years of follow-up. However thereafter the survival rate remains static for partial nephrectomy though it continues to decline for radical nephrectomy (Figure 1). This may be due to the smaller early tumours that are subjected for partial nephrectomy. The local recurrences remain low even after partial nephrectomy which is reassuring and hence it should be the preferred surgical option for appropriately selected patients.

In conclusion, papillary RCC has a worse prognosis than clear cell RCC which needs further study. Partial nephrectomy appears to be oncologically sound similar to radical nephrectomy. Adrenal gland involvement in RCC is low.

\section{Conflicts of interest}

There are no conflicts of interest.

\section{Funding}

No source of funding.

\section{References}

1. Siegal RL, Miller KD, Jemal A. Cancer statistics, 2018. CA Cancer J Clin 2018; 68: 7-30.

2. Cancer Incidence Data: Sri Lanka year 2014. National Cancer Control Programme, Sri Lanka. 2019.

3. Cancer Incidence Data: Sri Lanka year 2010. National Cancer Control Programme, Sri Lanka. 2016.

4. Balagobi B, Indika K, Samaraweera MCK, Wijayarathna KSN, Maddumage HP, Sutharshan K, et al. Risk factors of renal cell carcinoma in a cohort of Sri Lankan patients: A case-control study. J Cancer Res Therapeutics 2019; 15: S91-7.

5. Ratnatunga C, Goonewardena S, Anuruddha A. A Bibliography of publications relating to urological surgery in Sri Lanka 1887-2019. 2020; 16-8.

6. Kutikov A, Uzzo RG. The R.E.N.A.L nephrometry score: a comprehensive standardized system for quantitating renal tumour size, location and depth. J Urol 2009; 182: 844-8.

7. Moch Holger, Humphrey Peter A, Ulbright Thomas M, Reuter Victor E. World Health Organization Classification of Tumours of the Urinary System and Male Genital Organs, $4^{\text {th }}$ edition, IARC Press: Lyon, 2016; 12.

8. Kanayama Hiro-omi, Fukumori Tomohary, Fujimoto Hiroyuki, Nakanishi Hiroyuki, Ohyama Chikara, Suzuki Kazuhiro, et al. Clinicopathological characteristics and oncological outcomes in patients with renal cell carcinoma registered in 2007: The first large-scale multicenter study from the Cancer Registration Committee of the Japanese Urological Association. Int J Urol 2015; 22: S1-7.

9. Thorstenson Andreas, Harmenberg Ulrika, Lindblad Per, Holmstrom Benny, Lundstam Sven, Ljungberg Borje. Cancer characteristics and current treatments of patients with renal cell carcinoma in Sweden. Bio Med Res Int 2015; 2015: 456040.

10. Joshi Amit, Anand Amitesh, Prabash Kumar, et al. Kidney cancer demographics and outcome data from 2013 at a tertiary cancer hospital in India. Indian J Cancer 2017; 54: 601-4.

11. Sandra S, Martin J, Roos Frederik C, Frank B, Simon S, Christoph S, et al. Incidence and long-term prognosis of papillary compared to clear cell renal cell carcinoma - A multicentre study. Euro J Cancer 2012; 48: 2347-52.

12. Babu SC, Mianoni T, Shah PM, Goyal A, Choudhury M, Eshghi M, et al. Malignant renal tumour with extension to the inferior vena cava. Am J Surg 1988; 176: 137-9.

13. Hellsten S, Berge T, Lineli F. Clinically unrecognised renal carcinoma: aspects of tumour morphology, lymphatic and haematogenous metastatic spread. Brit J Urol 1983; 55: 166-9. 